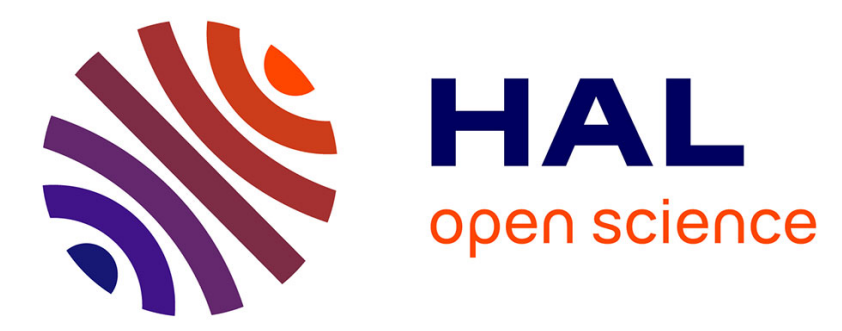

\title{
Fine-tuning and visualization of convolutional neural networks
}

Xiangnan Yin, Weihai Chen, Xingming Wu, Haosong Yue

\section{To cite this version:}

Xiangnan Yin, Weihai Chen, Xingming Wu, Haosong Yue. Fine-tuning and visualization of convolutional neural networks. 2017 12th IEEE Conference on Industrial Electronics and Applications (ICIEA), Jun 2017, Siem Reap, France. pp.1310-1315, 10.1109/ICIEA.2017.8283041 . hal-03467306

\section{HAL Id: hal-03467306 https://hal.science/hal-03467306}

Submitted on 6 Dec 2021

HAL is a multi-disciplinary open access archive for the deposit and dissemination of scientific research documents, whether they are published or not. The documents may come from teaching and research institutions in France or abroad, or from public or private research centers.
L'archive ouverte pluridisciplinaire HAL, est destinée au dépôt et à la diffusion de documents scientifiques de niveau recherche, publiés ou non, émanant des établissements d'enseignement et de recherche français ou étrangers, des laboratoires publics ou privés. 


\title{
Fine-tuning and Visualization of Convolutional Neural Networks
}

\author{
Xiangnan Yin, Weihai Chen*, Xingming Wu and Haosong Yue \\ School of Automation Science and Electrical Engineering \\ Beihang University \\ Beijing 100191, China \\ yinxn26808@126.com, whchen@buaa.edu.cn
}

\begin{abstract}
Image classification is a widely discussed topic in the field of computer vision. In recent years, with the application of Convolutional Neural Networks (CNNs), the state-of-the-art in this area has progressed rapidly. To yield a well performed CNN, the advanced GPU and large amount of training data are employed, thus training an entire $\mathrm{CNN}$ from scratch is difficult. In practice, fine-tuning a pre-trained $\mathrm{CNN}$ is a simple yet effective method to solve a target task. In this paper, we address on the issue of visualizing a fine-tuned $\mathrm{CNN}$, comparing with a small CNN trained from scratch on the same task, to explain how fine-tuning achieve such good performance.
\end{abstract}

\section{INTRODUCTION}

Traditional image classification algorithms have two main steps: (1) extracting features with hand crafted feature extractor, (2) setting the parameters of a trainable classifier. While the Convolutional Neural Network (CNN) is an end-to-end learning method, which means we don't need to design the features extractors ourselves, instead the algorithms can learn both the feature extractor and build a trainable classifier. In recent years, with the development of computer hardware, CNNs are widely used in image classification. However, the lack of enough training data and the long training time make it difficult to build a well-performed CNN from scratch. For this reason, training $\mathrm{CNNs}$ with less training data and computational cost becomes a popular research direction. Some researches indicate that with the increasing of the layers, the features extracted by the $\mathrm{CNN}$ are becoming more and more complex [1]. In convolutional networks, the first layers often specialize to detect simple features like edges, combination of edges, while the later layers detect higherorder combinations of these features like faces, feathers, whole objects or object parts according to the specific task. Based on this fact, the fine-tuning approach privileges the generic descriptors extracted from a pre-trained CNN, which helps to avoid training the entire network.

Some of the recent research efforts have been devoted to visualizing the image features detected by each layer of a network. One way to visualize the role played by a layer in detection is by finding an image that activates many neurons of this layer. Inspired by the procedure of [2], [3], with a trained network, we don't modify it's parameters, instead, we modify the input image by performing gradient ascent to maximize the target layer's output. In this way, features extracted by the target layer could be visualized.

\footnotetext{
*Corresponding author: whchen@buaa.edu. cn
}

In this paper, we start with training a small $\mathrm{CNN}$ on Asirra CAPTCHA [4] to solve the problem of cat vs. dog classification, which reaches an accuracy of 0.82 as a baseline. Then we fine-tune the architecture and the weights of VGG16 [5] toward the same classification task. Our fine-tuning approach reaches an accuracy of 0.95 , which is 0.12 higher than the traditional state-of-art algorithm [6]. At last, with the visualization approach mentioned above, we visualize different layers of the baseline $\mathrm{CNN}$ and the finetuned CNN. By visualizing differences between the output layers of two CNNs, we conclude an intuitive explanation on how fine-tuning could be such efficient and effective.

\section{A. Related work}

Since the appearance of the CNNs, several approaches for understanding and visualizing CNNs are developed. Visualizing the layers activations during the forward pass is the most straight-forward technique. But as the network goes deeper, the activations tend to become more and more sparse, some of the activation maps may stay zero no matter how we change the inputs, which indicates the dead filter. Another common strategy is to visualize the weights of the filter. Usually, a well-trained network have filters with more smooth patterns, and a network not trained enough have more noisy filters. However, those two strategies above are not intuitive enough in explaining the features learned by neural networks. To show exactly what the neuron is sensitive about, some researchers try to link the feature space of a target neuron back to the input space, they feed large amount of images to the network, and look for the images which maximally activate the neuron [7], [8], [9], the reason behind this idea is that a pattern to which the unit is responding maximally could be a good first-order representation of what a unit is doing [10]. However, the method only shows the patches in feature space alone the nature basis, while [11] found that with any random direction, the retrieved images show also similarly explainable semantic properties, which suggests that the method cannot show the essential property of a neuron. Some methods embed the high-dimensional feature map into two dimensions, while preserving the pairwise distances, which can roughly visualize the topology of feature space, among various embedding algorithms, tSNE [12] is the most commonly used. Deconvolution [8] the feature activations back to the input pixel space can also get an intuitive explanation on the features learned by 


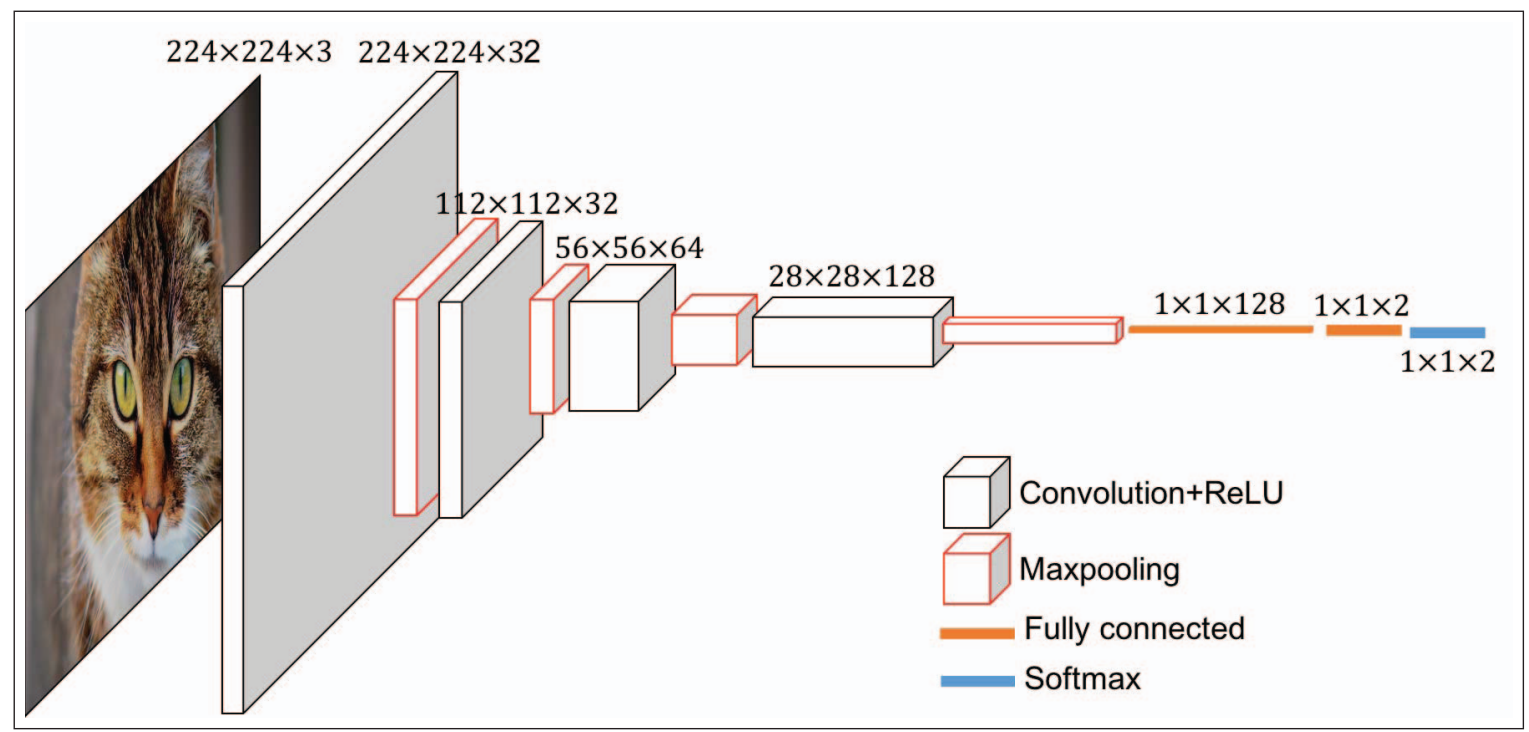

Fig. 1. The architecture of our baseline CNN. The input is a $224 \times 224 \times 3$ color image. This is convolved with 32 different 1 st layer filters, each of size $3 \times 3$, using a stride of 1 in both $\mathrm{x}$ and $\mathrm{y}$ direction, the boarder mode is 'same'. The result feature maps are then: (i) passed through a ReLU function, (ii) max pooled within $2 \times 2$ regions, with stride 2 . Similar operations are repeated four times. Then, the next two layers are fully connected, between which a dropout operation (rate 0.5) is included to reduce overfitting (which is not shown in figure). The result of full-connected layers is passed to a two-class softmax function, outputting the probability of each class i.e. cat and dog.
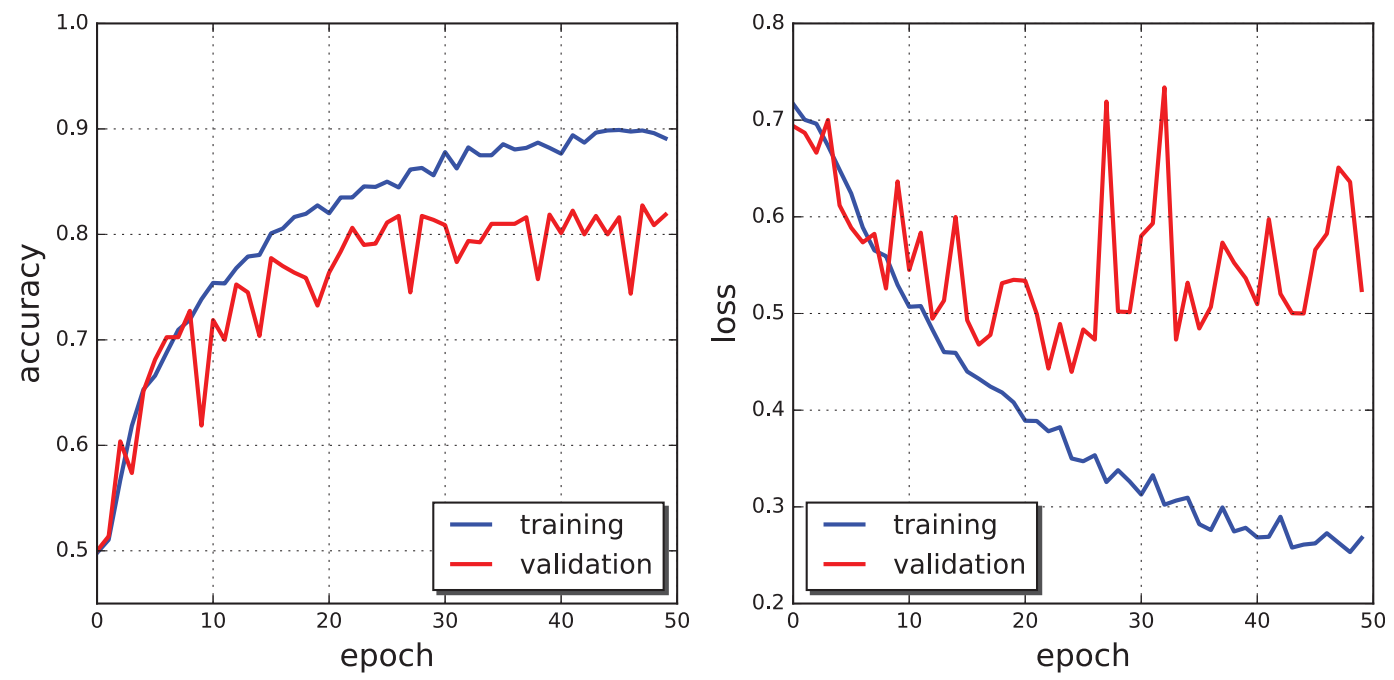

Fig. 2. Accuracy and loss of the training process.

each layer. Despite the great achievement in [8], the author only visualizes the individual filter of a layer, but there exists hundreds of filters in each layer, to give a general visualization of the whole layer, our visualization approach is deployed.

\section{TRAINING CNNS}

To illustrate the power of fine-tuning, we will train two CNNs. The first one has a small size thus can be trained from scratch with little data, while the second one is trained by fine-tuning the VGG16 [5], both of the CNN are trained on the same data base (2000 images for training and 400 images for validation).

We trained our CNNs on Keras[13] and Tensorflow [14] framework during experiments. To prevent overfitting, we employed the real-time data augmentation module of Keras[13], which could randomly resize, flip and shear the input images while training.

\section{A. Training baseline $C N N$}

In this section, we introduce the training of a small $\mathrm{CNN}$, which serves as a baseline. The architecture of our baseline CNN is illustrated in Fig 1.

Fig 2 shows the evolution of the accuracy and the loss with respect to the training epoch. We could figure out that after 50 epochs of training, the accuracy on the validation set stays at around 0.81 . However, the accuracy on the training set is about 0.9 higher than the validation set, and the loss of validation set diverges while training epoch increases, this is the consequence of overfitting, which is due to the little 


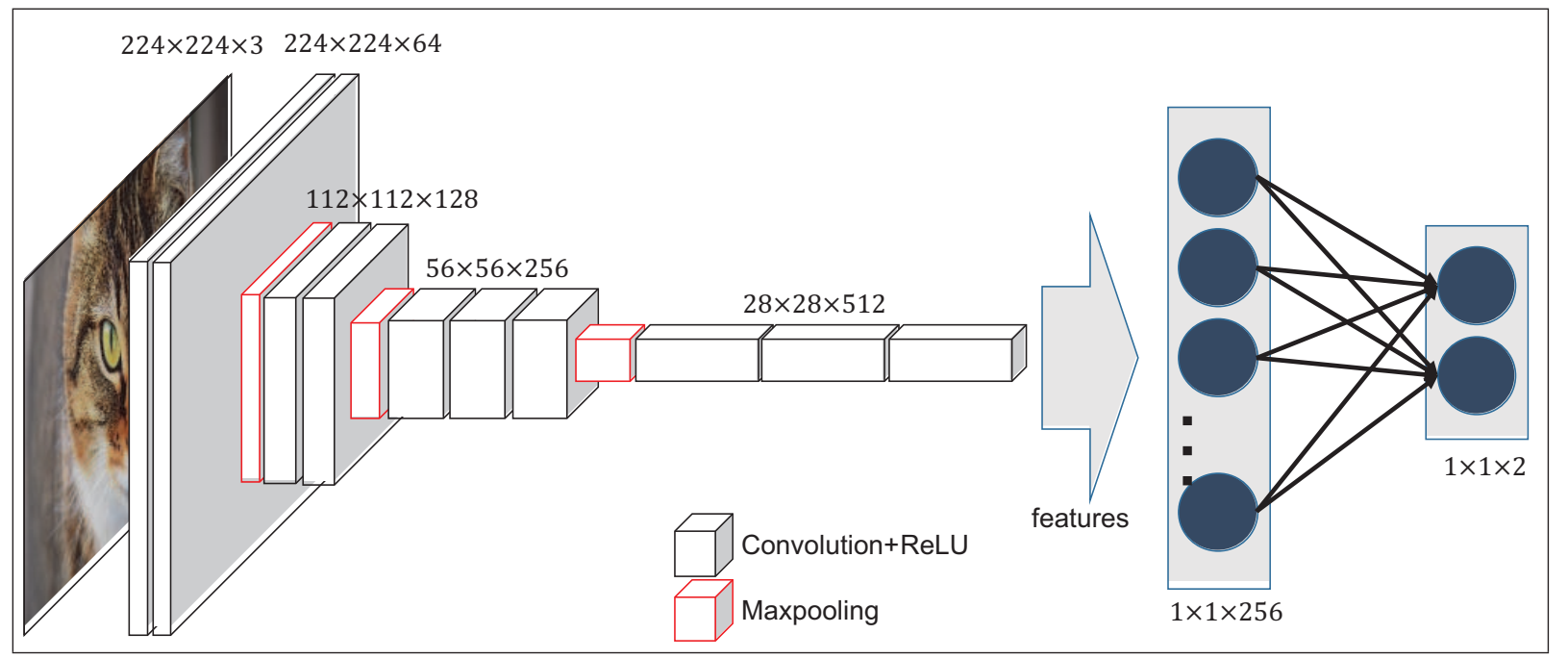

Fig. 3. The architecture of fine-tuned CNN. Left: four convolutional blocks of pre-trained VGG16, which serves as an feature extractor on the first term training. Right: self-defined fully connected networks, which composes one hidden layer with 256 neurons and one output layer of two neurons, dropout of 0.5 is added between two layers, softmax is used as an activation function of the last layer. While fine-tuning, only the last two convolutional blocks and the self-defined layers are trainable.
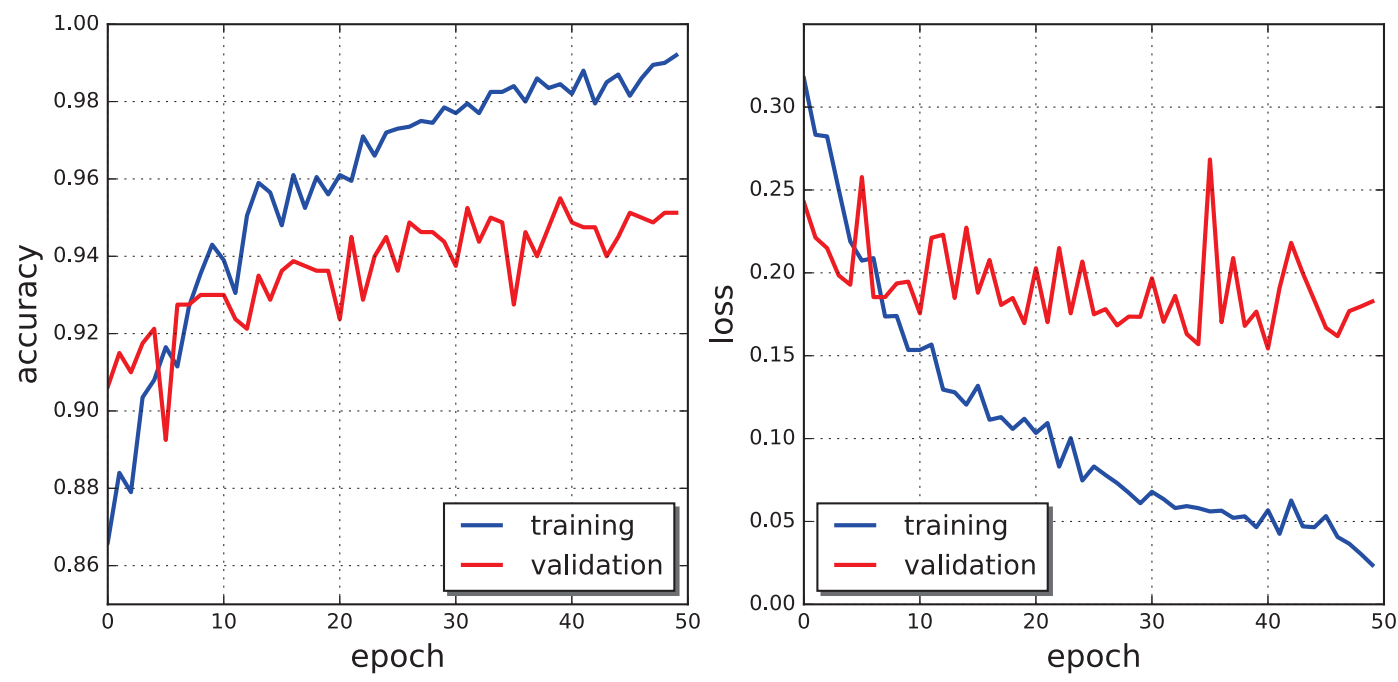

Fig. 4. Accuracy and loss of the fine-tuning process.

training data.

\section{B. Fine-tuning the VGG16}

In this section, we make use of VGG16 [5] pre-trained on ImageNet dataset.

In the fine-tuning technology, the first $n$ layers are initialized by a pre-trained $\mathrm{CNN}$, and the top layers are self-defined according to our target task and are randomly initialized. For the purpose of protecting the pre-trained weights not to be wrecked, one can either renew the weights of the entire neural network in a small learning rate, or fix the earlier layers and only fine-tune the top layers of the network. To balance the difference of learning rate for the pre-trained layers and the self-defined layers, our approach is composed of two terms training as follows:

- In the first term, we apply the method of traditional transfer learning, the fully-connected layers of VGG16 are removed, the rest layers (see Fig 3 left) are used as fixed feature extractor. With the features extracted by the convolutional layers, we roughly train a small selfdefined network with two outputs (Fig 3 right) by using ADAM adaptive optimization[15].

- In the second term of training, we add the roughly trained network to the top of the convolutional layers of VGG16. By a SDG of small learning rate (1e-4), we update the parameters of the last two convolutional blocks of VGG16 and the self-defined layers.

The change of accuracy and loss during training is shown in Fig 4. The figure shows that after 50 epochs training, the accuracy on the training set is about 0.4 higher than the validation set, and the loss of the validation set is converged between 0.15 and 0.20 . Comparing to the baseline CNN, the problem of overfitting is much more reduced. 
The following table shows the result of our training. It's clear that the second term's performance is better than the first term, and both of them outperforms the baseline CNN trained from scratch. Which demonstrates the power of a pre-trained network in feature extraction.

\section{TABLE I}

SUMMARY OF THE PERFORMANCE

\begin{tabular}{|c|c|c|c|}
\hline & Baseline & First term & Second term \\
\hline Training Accuracy & 0.8910 & 0.8895 & 0.9920 \\
\hline Validation Accuracy & 0.8188 & 0.8900 & 0.9512 \\
\hline Training Loss & 0.2678 & 0.2472 & 0.0237 \\
\hline Validation Loss & 0.5243 & 0.2950 & 0.1828 \\
\hline
\end{tabular}

\section{VisUALIZATION}

With our fine-tuning approach in the previous section, we made significant progress in classification accuracy. But how can a pre-trained network be such powerful, in order to understand the operation of fine-tuning, we need to interpret the feature activity in the hidden layers, which involves the visualization of network layers.

The idea of visualization is as follows: given a network of $N$ layers, starting from an input image, we would like to modify the input image pixels to maximize the output of a target layer $l<N$. The reason is simple: a pattern which the layer is responding maximally could be a good representation of what the layer is doing. In equation (1) and (2), the idea is generalized to an optimization problem. Let $\theta$ denote the parameters of a network, and let $x$ be the input image, $x^{(i)}$ be the feature maps of layer $i$ of the $\mathrm{CNN}$, the target is to look for $x^{*}$ such that maximize the $f\left(x^{(i)}\right)$, where $f$ denotes an objective function. While visualizing the hidden layers, we set it to the $L^{2}$ norm of $x^{(i)}$, later we change it to other forms to find an input that maximizes a specific class.

$$
\begin{gathered}
x^{(i)}=l_{i}(\theta, x) \\
x^{*}=\arg _{x} \max f\left(x^{(i)}\right)
\end{gathered}
$$

This is a non-convex optimization problem, but we can try to find a local maximum by performing gradient ascent on the input data. In concrete terms, the algorithm contains the following steps:

1) Set the objective function of the target layer.

2) Calculate the derivative of objective function with respect to the input image

3) Renew the input image by gradient ascent using function (3)

$$
x:=x+\lambda \frac{\mathrm{d} f}{\mathrm{~d} x}
$$

After several steps of gradient ascent, the patterns which mostly activate target layer should be visualized on the input image. Algorithm 1 explains our approach in detail:

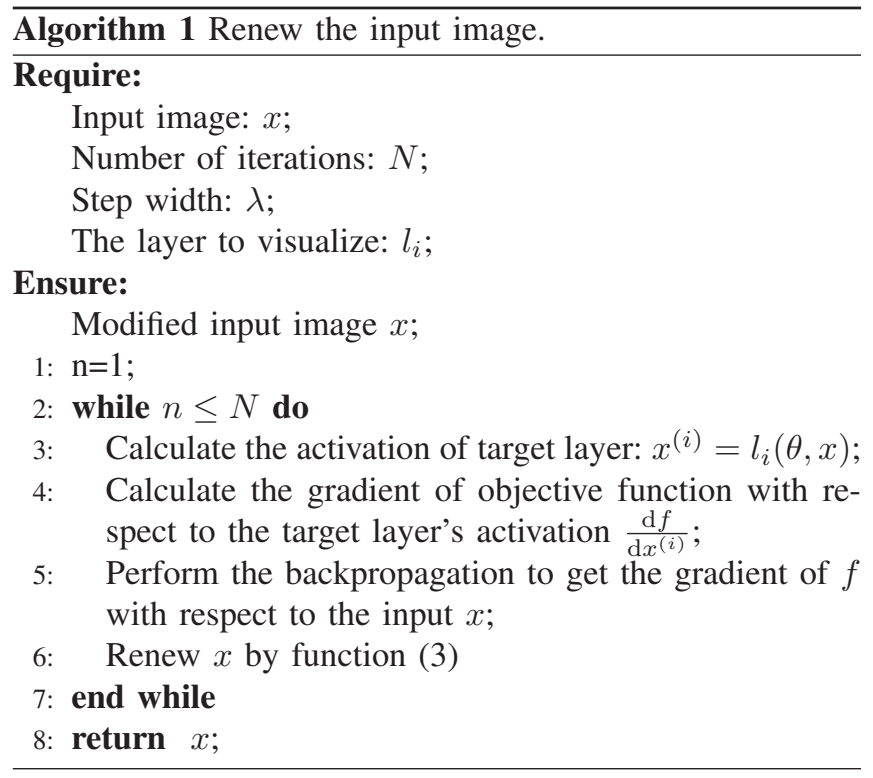

\section{A. Visualization of hidden layers}

To visualize the hidden layers of a $\mathrm{CNN}$, we set the objective function as equation (4) and equation (5) shows the jacobian of the objective function with respect to the target layer's activation.

$$
\begin{aligned}
& f\left(x^{(i)}\right)=\frac{1}{n}\left\|x^{(i)}\right\|_{2} \\
& \frac{\mathrm{d} f\left(x^{(i)}\right)}{\mathrm{d} x^{(i)}}=2 \times x^{(i)}
\end{aligned}
$$

Based on equation (5) and the chain role, we back propagate to the input layer, and the gradient of $f$ with respect to input image $x$ can be obtained. Instead of feeding the network with noise image as in [2], we feed the network with images which have the highest score in each class. The reason is simple, having a high classification score means that the image is close to the local maxima, thus our gradient ascent would more likely to emphasize some of the image features, rather than creating the features from a noise image, this could make it easier to investigate what the target layer interests about.

In our experiment, we visualized the layers of the baseline CNN Fig 5, and the fine-tuned VGG16 Fig 6. The left most image of each row is the original image, which has the highest score of it's class. From left to right, the layers goes from base to top.

In Fig 6, we can easily conclude that when the layer goes higher, the features extracted becomes more and more complex. The base layers emphasize only simple patterns like color blobs, edges, while the top layers emphasize hair, eyes and noses. With the baseline model, same tendency could be concluded, however, the features extracted from the top layers are not explicable. This is due to the poor ability of feature extraction compared to VGG16. 


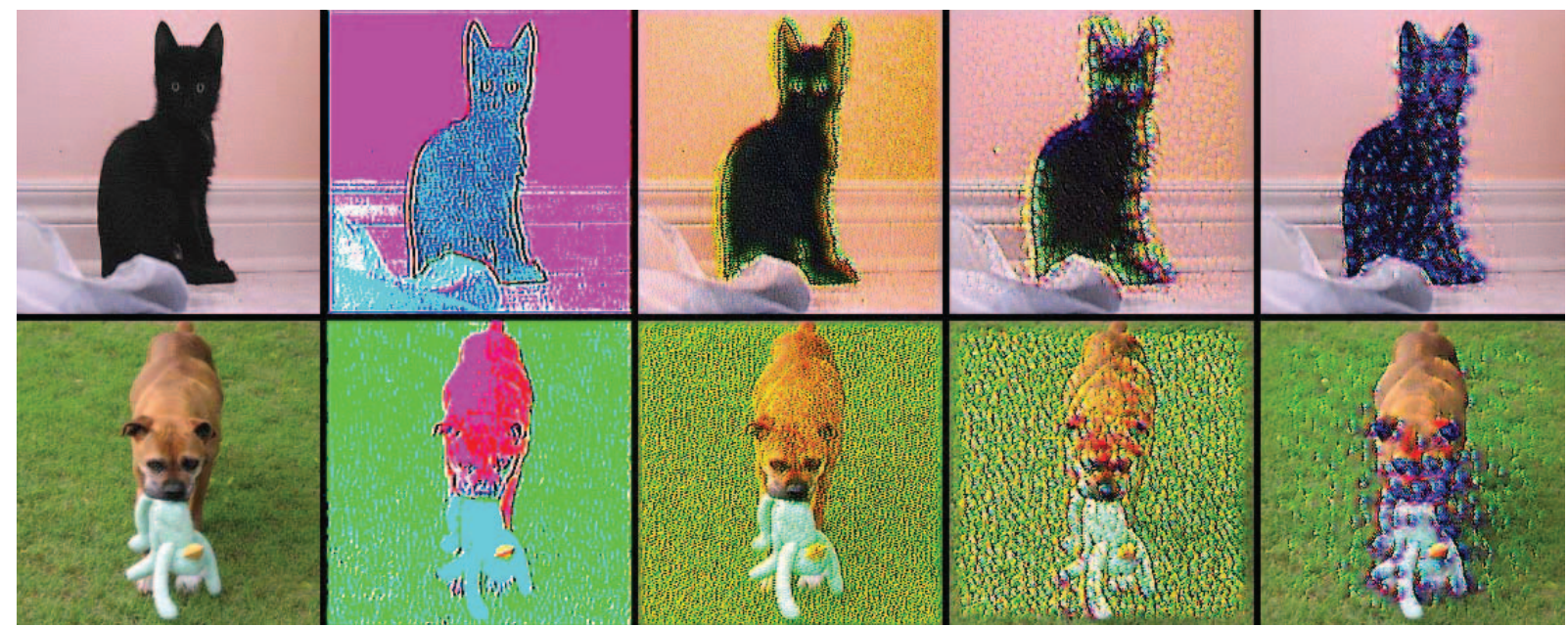

Fig. 5. Layers of baseline CNN. All the convolutional layers are visualized

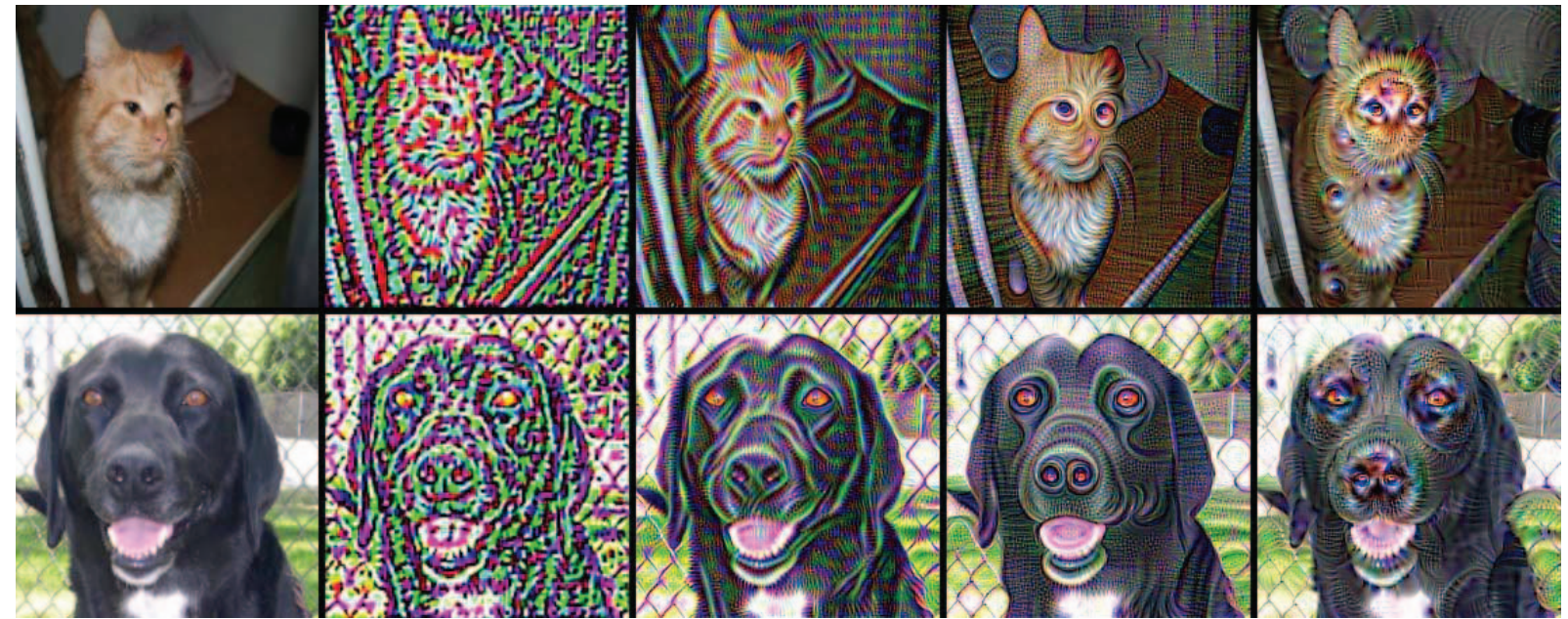

Fig. 6. Layers of fine-tuned CNN. We visualized the first layers of convolutional blocks from 2 to 5

\section{B. Visualization of the final layer}

To investigate what the entire CNN learns about, we would like to visualize the output layer of the network. Since our output activation is softmax, in our two classes classification it has the following form:

$$
\sigma\left(z_{i}\right)=\frac{\exp \left(z_{i}\right)}{\exp \left(z_{1}\right)+\exp \left(z_{2}\right)} \quad i=1,2
$$

The outputs are probabilities between 0 and 1 , considering the precision of the computer (the floating point number precision is limited), in our experiment, instead of maximizing each outputs of the last layer, we deal with the second last layer. Let $z_{1}, z_{2}$ denote two activations of the second last layer, which represent cat and dog respectively. If the image looks more like a cat, $z_{1}$ should be much bigger than $z_{2}$, in the contrary, $z_{2}$ should be much bigger than $z_{1}$. Our objective function could simply be the difference between $z_{1}$ and $z_{2}$, $z_{1}-z_{2}$ for cat and $z_{2}-z_{1}$ for dog.

Fig 7 shows the patterns of cats and dogs extracted by the second last layers of our two trained models. We find that, with the fine-tuned network, for cat images, patterns like hair, ears, and body arcs are emphasized, for dog images, noses, eyes are emphasized. Those differences between dogs and cats are consistent with our life experience. While with the baseline $\mathrm{CNN}$, we can only state that there exist changes locate on the faces of dogs and cats, but no explainable semantic patterns could be figured out.

\section{ANALYSIS}

A network pre-trained on a large dataset has prior knowledge in the task of image classification, without training, it "knows" already what kind of patterns should be extracted. While fine-tuning, as the layer goes deeper, the latter layers combine the features extracted from the former layers to form more complex patterns, by slightly modifying the top layers' weights, such combination of features becomes more and more suitable towards our classification task, finally, we could extract explainable semantic features from the input images. This is the reason why fine-tuning outperforms our baseline CNN. 

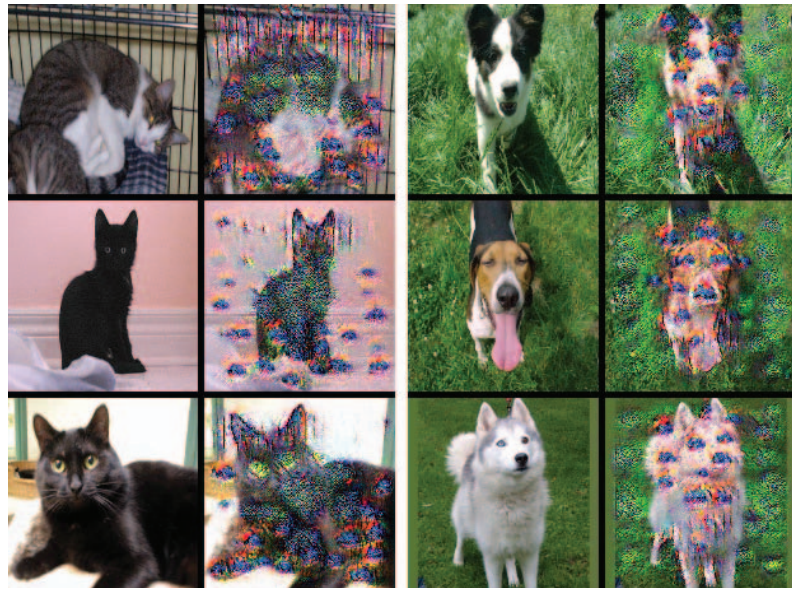

(a) Baseline CNN
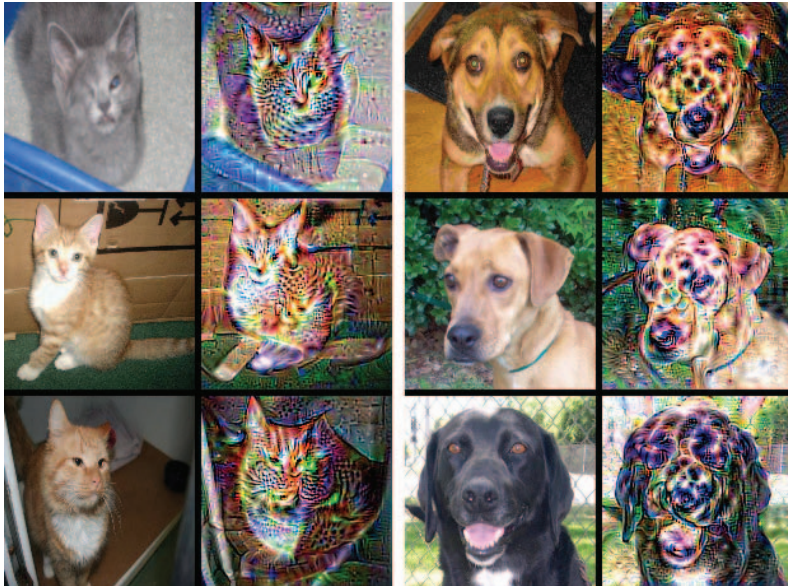

(b) Fine-tuned VGG16

Fig. 7. Second last layers of two trained models. On the left is the baseline model, and on the right is the fine-tuned VGG16 model. For each network, we choose the top 3 scored images of each class as input.

In some sense, the learning process of a $\mathrm{CNN}$ is just like human beings. To learn some thing new, people have basis (fine-tuning a network) learn faster (training network from scrath) than people without basis.

\section{CONCLUSIONS}

In this paper, two learning strategies are employed toward the same classification task. We compared the performance of the fine-tuned $\mathrm{CNN}$ with the CNN trained from scratch. A novel method of visualizing target layers of a $\mathrm{CNN}$ is proposed. By visualizing the layers of two CNNs, our research gives an intuitive explanation on the efficiency of the fine-tuning strategy.

\section{ACKNOWLEDGMENT}

This work has been supported by National Nature Science Foundation of China under the research project 61620106012,61573048 and 61603020.

\section{REFERENCES}

[1] Yosinski J, Clune J, Bengio Y, et al. "How transferable are features in deep neural networks?," In Advances in neural information processing systems, pp. 3320-3328, 2014.

[2] Simonyan K, Vedaldi A, Zisserman A. (2013) "Deep inside convolutional networks: Visualising image classification models and saliency maps," arXiv preprint arXiv:1312.6034.

[3] Alexander Mordvintsev, Christopher Olah, and Mike Tyka. (2015, June 20) Inceptionism: Going deeper into neural networks [Online]. Available: https://research.googleblog.com/2015/06/inceptionismgoing-deeper-into-neural.html

[4] Elson J, Douceur J R, Howell J, et al. "Asirra: a CAPTCHA that exploits interest-aligned manual image categorization," In ACM Conference on Computer and Communications Security, 2007, vol. 7, pp. 366-374.

[5] Simonyan K, Zisserman A. (2014) "Very deep convolutional networks for large-scale image recognition," arXiv preprint arXiv:1409.1556.

[6] Golle P. "Machine learning attacks against the Asirra CAPTCHA," In Proceedings of the 15th ACM conference on Computer and communications security, 2008, pp. 535-542.

[7] Girshick R, Donahue J, Darrell T, et al. "Rich feature hierarchies for accurate object detection and semantic segmentation," In Proceedings of the IEEE conference on computer vision and pattern recognition, 2014, pp. 580-587.
[8] Zeiler M D, Fergus R. "Visualizing and understanding convolutional networks," In European conference on computer vision, Springer International Publishing, 2014, pp. 818-833.

[9] Goodfellow I, Lee H, Le Q V, et al. "Measuring invariances in deep networks," In Advances in neural information processing systems, 2009, pp. 646-654.

[10] Erhan D, Bengio Y, Courville A, et al. "Visualizing higher-layer features of a deep network," In Technical report, University of Montreal, 2009, 1341:3.

[11] Szegedy C, Zaremba W, Sutskever I, et al. (2013) "Intriguing properties of neural networks," arXiv preprint arXiv:1312.6199.

[12] Hinton, G. E. "Visualizing High-Dimensional Data Using t-SNE," Vigiliae Christianae, 9(2):2579-2605, 2008

[13] Chollet, Franois. (2015) Keras: Deep learning library for theano and tensorflow [Online]. Available: https://keras.io

[14] Abadi M, Agarwal A, Barham P, et al. (2016) "Tensorflow: Largescale machine learning on heterogeneous distributed systems," arXiv preprint arXiv: 1603.04467.

[15] Kingma D, Ba J. Adam (2014) "Adam: A method for stochastic optimization," arXiv preprint arXiv:1412.6980. 